\title{
A New Method of Fingerprints' Singular Points Detection
}

\author{
Zhang Zhe, Liang Yuanyuan and Liu Xiangjiao \\ Software College, Nan Yang Normal University, Henan 473000, China \\ Yuan_hnlw@126.com
}

\begin{abstract}
This paper proposes a new method of fingerprint singular points searching algorithm based on fingerprint orientation maps mainly. In these orientation maps, mathematical morphology theory and operations are used to search the fingerprint's singular points in view of the phenomenon that the change of ridges' direction at the points is the fastest in the whole fingerprint image and the directions of finger ridges which surround the points are symmetric with each other. For obtaining more accuracy result, before obtain the orientation maps, we use db3 wavelet technology to remove the most of fingerprint images' noises in this paper. For the same reason, before the main mathematical morphology operations, we first obtain fingerprint images' binary image models based on the db3-denoise image, and use these models to remove the disturb directions of the image background secondly. Experiments indicate this method is easy to understand, achieve and has a good robustness for the fingerprint's translation and deformation.
\end{abstract}

Keywords: Fingerprint Matching Algorithm; Mathematical Morphology; Fingerprint Singular Points' Searching Method

\section{Introduction}

As we known, fingerprint's singular points are the most representative features of the fingerprint ridges. They are uniqueness, having obvious characteristics and often in a special location just like the center of the fingerprint. So they are easily to be found and to be as a reference. For these reasons, the vast majority of the fingerprint matching algorithms are based on the singular points' matching firstly, such as the fingerprint point pattern matching methods. So the accurately of finding the fingerprints' singular points is the most important part of the fingerprint's matching algorithm.

Currently, the more common ways of detection for singular points are as follows: Poincare index way [1]; method based on templates and thinning image[2]; method based on the features of orientation map [3-4]; method based on ridge curvature's calculation [56]. In recent years, some new theories and models [7] are used in this field, and people tend to coalesce more features of fingerprint [8] and more method to get accident result. As a whole, there are still many problems waiting to be solved. Just like: large in computing capacity, higher quality requirements of images, lower in accuracy, and so on.

Aiming at these problems, this paper proposes a new method that is still handled on orientation map to find the singular points by mathematical morphology method, and using ridges' symmetrical characteristic which near the central point of the fingerprint. The db3-wavelet and some other image processes are used to avoiding the noises' influence which just cost a little time, but make the whole algorithm have a better accuracy. The result shows that this method is good at the method of Fingerprints' Singular Points Detection.

As shown in figure 1, the singular points are divided into two types: center point and delta point [1]. They have big difference in fingerprint ridges' directions which go around them. 


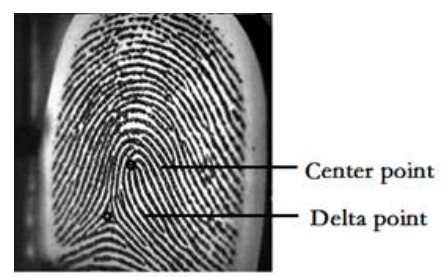

\section{Figure 1. A Example of Singular Points in Fingerprint Image}

\section{Introduction of Algorithm Procedures}

As a whole, the algorithm of this paper has several essential procedures which are made up by 5 big steps mainly. Each big step only does one thing, and all of the 5 steps must to be done in an order. In addition, each big step is also having several small steps which still have an order. And we will introduce them in the follow figure 2 more clearly. In the following passage, we introduce them simply:

Step1: Use the db3 wavelet method to remove the original fingerprint images' noises.

Step2: Obtain fingerprint image orientation maps based on the images gained by step1.

Step3: Obtain binary fingerprint models based on the images gained by step1.The binary models are still images which have the same size as the original images.

Step4: Remove the fingerprint's border noises by means of performing a bitwise AND on 2 images from step2 and step3.

Step5: Search singular points by using some mathematical morphology operations which we called main algorithms.

At the end, we gain the information of the singular points. In all 5 steps, we can know that each step is easy to understand and for writing a program. And each of them is low in time consuming.

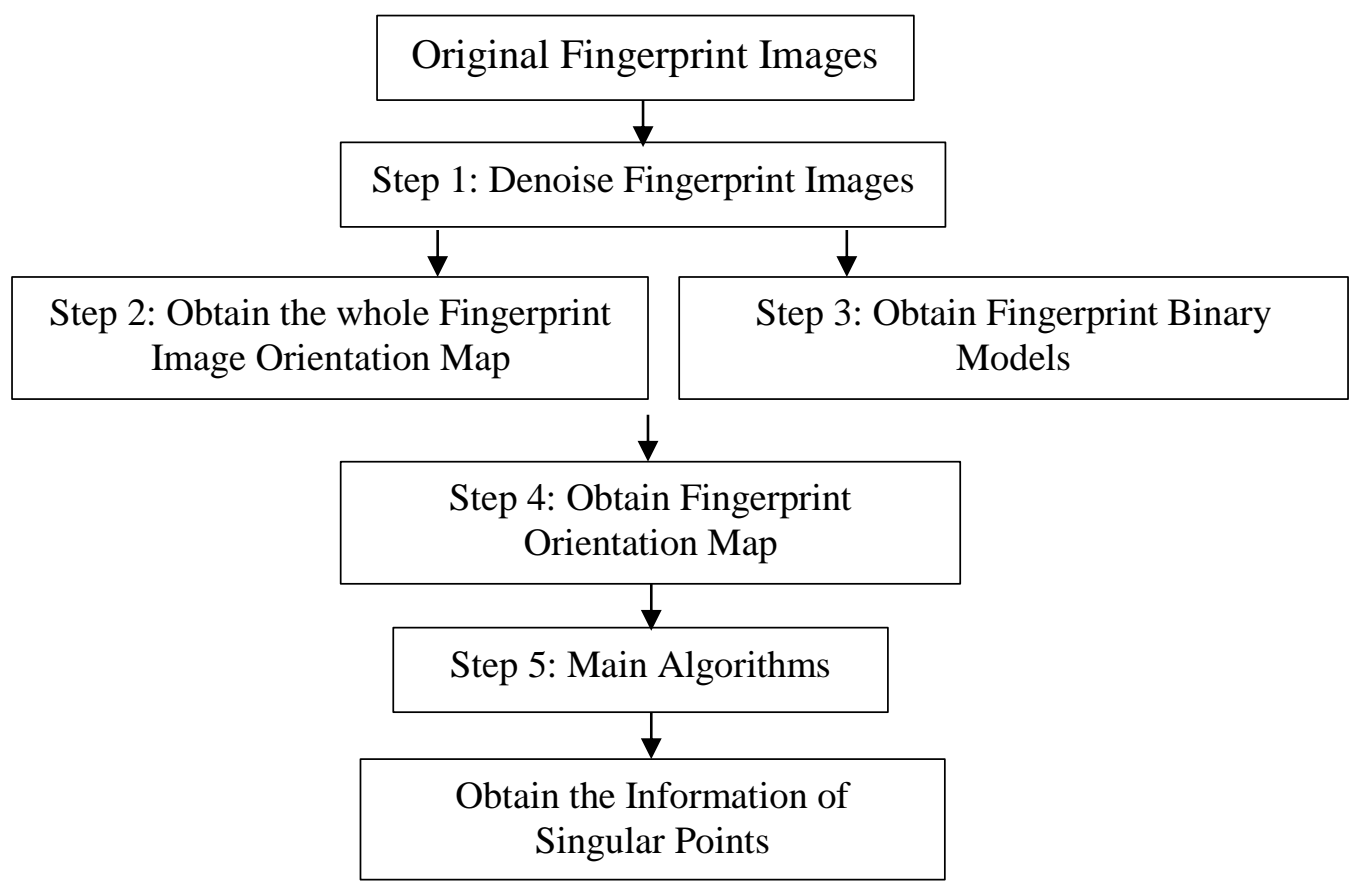

Figure 2. The Whole Algorithm Procedures

\section{The Theory of Db3 Wavelet}

As we known, wavelet is one kind of frequency domain method which has the multi resolution characteristic [9], freedom in selection of wavelet bases [9], a high running 
speed, and so on. Because of these reasons, the method is having more and more applications in the signal and image deionizing processing field. In this paper, we use db3 wavelet to decompose the images on the one level, and then reconstruct the image to make the fingerprint images more clearly before obtaining orientation map and obtain binary image models.

Db3 wavelet is one of the $\mathrm{dbN}$ wavelet bases which were created by famous scholar Ingrid Daubechies [9]. This wavelet basis has no analytic expression but better expansibility which can solve boundary problem flexibly.

Figure 3 is db3 wavelet graphs drew by matlab software. The above graph is the scale function, and the below graph is the wavelet function.

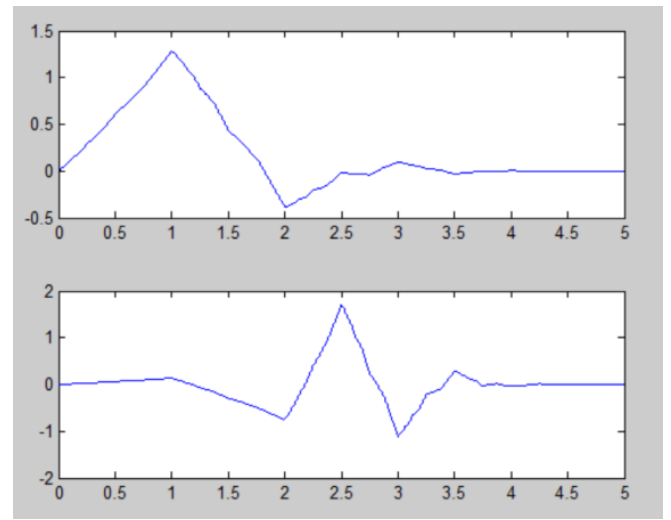

Figure 3. A Graphic of Db3 Wavelet Bases

\section{Introduction of Mathematics Morphology}

The mathematics morphology is a very important nonlinear theory on image processing. The scholars of G. Matheron and J. Serra in Paris Mining Institute created the theory in 1964 [10]. The research effort has focused on the shape, size and topological relation of the object. Its theoretical foundation is set theory, grid pattern, topology and probability theory.

There are many types of operation in mathematical morphology. The erosion, dilation, open, close operation are the four fundamental operations. With the exception of them, mathematical morphology also has hit-miss, top-hat, bottom-hat and other operations [10]. Open and close operations are opposite to each other, and all based on the composition of erosion and dilation operations. The other operations are based on the different composition of the four fundamental operations. Initially, the theory can only deal with binary images; now they can deal with gray and even color images. The field that the theory devoted to become broader and the problem become more complicated.

The process of morphology operation need use auxiliary mathematical set which we called morphology structural element. The structural element is often smaller than object in size. There are many types of morphology structural element which have regular and irregular shape. The ways of forming them are also different. Classically, the sample shape of structural element is easier than the complete one. The figure 4 shows some common shape of morphology structure element: circle, square, rhombus, disk, and line and dotted pairs. They are all in perfect geometry status, but in digital computer status they are expressed as imperfect matrix form.

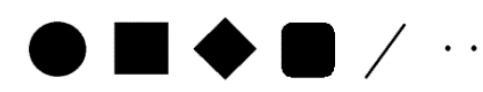

Figure 4. Six Common Morphology Structure Elements 
The expansion and corrosion of mathematical morphology is introduced below. In binary images, the expansion and corrosion operation can be explained simplify as the union and intersection of the object and structure element. But in gray and color images, they can be explained as the maximum and minimum operation of the object and structure element.

Let $\mathrm{A}$ and $\mathrm{B}$ are 2 sets of integer space $\mathrm{Z}$. A is the object. B is the structural element. The Erosion of $\mathrm{B}$ to $\mathrm{A}$ is defined as follows [11]:

$\emptyset$ is empty set. $\widehat{B}$ is reflection set of B.

$$
\mathrm{A} \oplus \mathrm{B}=\left\{\mathrm{z} \mid(\widehat{\mathrm{B}})_{z} \cap A \neq \emptyset\right\}
$$

The Dilation of $\mathrm{B}$ to $\mathrm{A}$ is defined as follows [11]:

$$
\widehat{\mathrm{B}}=\{\omega \mid \omega=-b, b \in B\}
$$

$$
\mathrm{A} \ominus \mathrm{B}=\left\{\mathrm{z} \mid(\mathrm{B})_{\mathrm{z}} \subseteq \mathrm{A}\right\}
$$

The $\mathrm{B}$ hit $\mathrm{A}$ is defined as follows [12]:

$$
B \cap A \neq \varnothing
$$

The $\mathrm{B}$ miss $\mathrm{A}$ is defined as follows [12]:

$$
\mathrm{B} \cap \mathrm{A}=\varnothing
$$

In the figure 5 , we use a $3 \times 4$ square as the object which shows in picture a). And a three-pixel short line as the structure element which shows in small picture b). For ease of illustration, the short line and the enlarged area are in gray color which should be having the same color with the object. The picture c) is a) expanded by b), and the gray pixels are just the expansion region. The picture d) is a) corroded by $b$ ).
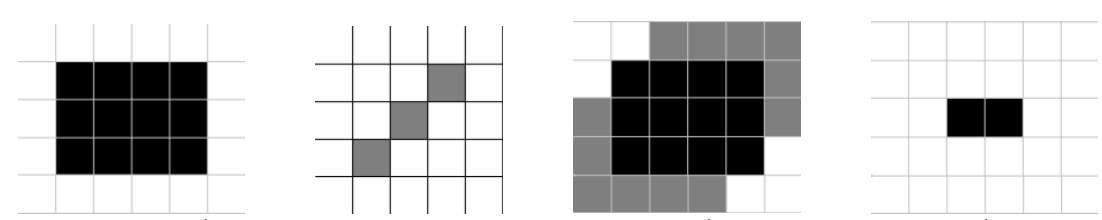

a) Original Image b) Morphology Structure Element c) Erosion Image d) Dilation Image

Figure 5. The Results of Erosion and Dilation Operation

The figure $6 \mathrm{~b}$ ) shows the hit operation on a), the structure element is also figure $5 \mathrm{~b}$ ). We can know that each original object transform into a point.

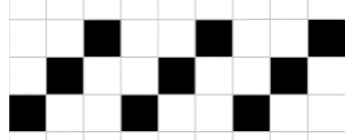

a) Original Image

b) Image after Hit Operation

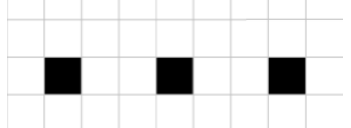

Figure 6. The Result of Hit Operation

\section{The Theory of Searching Singular Points}

The singular points of fingerprint image have many characteristics. The main characteristics we used in this paper are as follows: First, the change of ridges' direction is the fastest in the whole fingerprint image. Second, the ridges around the singular points have the characteristic of Symmetric on the basis of a symmetry axis, shows in figure 7 . From these two characteristics we can determine the coordinate values of the singular points accurately and exclusively.

The figure 7 shows there have 6 common types of fingerprint simple graphs: loop type, whorl type, arch type, double loop type, spiral whorl type, triradius type [13]. The figure 7 also shows that the four types of fingerprint's singular points are just at the junction of symmetry axis and the maximum-flexion ridge except the whorl type 
and the double loop type fingerprint. But these two types' center points are on the symmetry axis too.

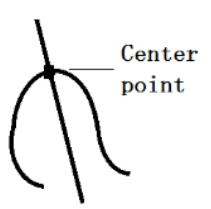

a) LoopType<smiles>CCCCCC(C)CC(C)CCCC</smiles>

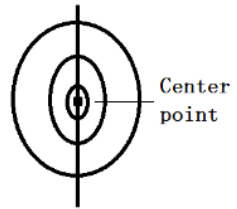

b) Whorl Type

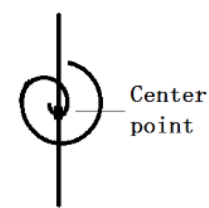

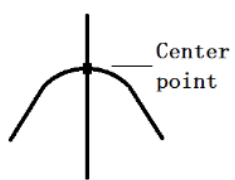

c) Arch Type

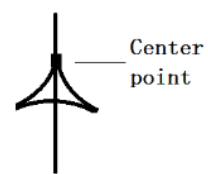

d) Double Loop Type e) Spiral Whorl Type f) Triradius Type

\section{Figure 7. Six Common Fingerprint Simple Graphs}

We can obtain two points at the whorl type fingerprint and the double loop type at the junction of symmetry axis and the maximum-flexion ridges. We find that the center point is just at the half way of these two points just like the figure 8 shows. So we can calculate the core point from the information of them. In this paper, we obtain the singular point from the orientation map of fingerprint image. As we known, the orientation map has all the direction information of fingerprint. So the method is feasible.

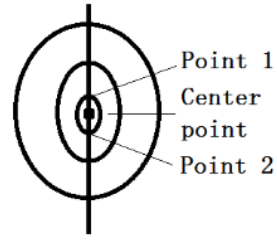

a) Whorl Type

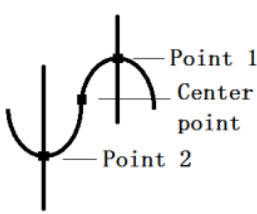

b) Double Loop Type

\section{Figure 8. Relationship of These Three Points}

\section{Step1: The Denoising Operation of Fingerprint Images}

In the first step, we use the db3 wavelet technology to remove the fingerprint image noises. We can know that most of the noises are at the background of the images. The noises between the fingerprint ridges and the shadow on the top of the image have no much bad influence on the subsequent operation. So the rest of noises are divided into two types:

1) The obvious edges of the image in black color which we can know from figure $10 \mathrm{a}$ ), can't be removed by the wavelet operation. So we will use the binary model which we obtain in the following steps to remove the disturbance of them.

2) Between the black edges and the fingerprint, there have some French grey punctate points with different shapes and sizes. And they are not very obvious in the 4 small pictures. We can reduce the color of them by adjusting the wavelet coefficient.

The figure 9 shows the 4 small images after one level db3 wavelet decomposition. The first image is the low frequency component which is similar to original image. The second image is low frequency in horizontal and high frequency in vertical, so it shows the horizontal edges of original image. The third image is just opposite to the second image. The fourth image is high frequency which shows the diagonal edges. The denoised image shows in figure $10 \mathrm{~b}$ ). 


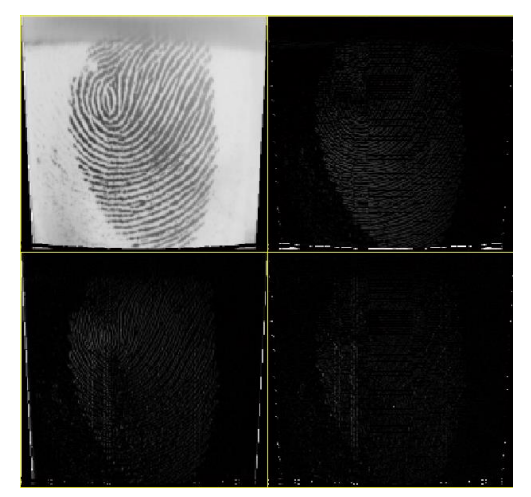

Figure 9. One Wavelet Decomposition for Fingerprint Image

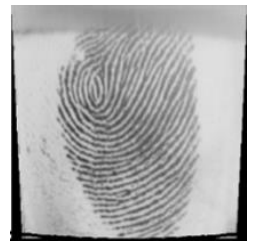

a) Original Image

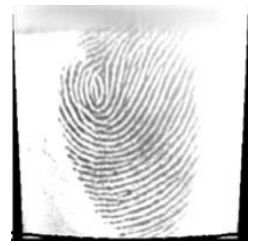

b) The result Image

Figure 10. The Original Image and Result Image

\section{Step2: The Obtaining of Fingerprint Orientation Map}

From above, we know the orientation map is the main carrier of searching for singular points in this paper. The algorithm of this big step is divided into three proceeds. And they must to be executed in a sequence.

First, we use $3 \times 3$ Sobel operators [14](Sobelx and Sobely, shows in (6)) which have two directions (horizontal and vertical) to dispose the image we gain from step1. The expressions of these 2 operators are as follows:

$$
\text { Sobelx }=\left[\begin{array}{ccc}
1 & 0 & 1 \\
2 & 0 & 2 \\
1 & 0 & 1
\end{array}\right] \quad \text { Sobely }=\left[\begin{array}{ccc}
1 & 2 & 1 \\
0 & 0 & 0 \\
-1 & -2 & -1
\end{array}\right]
$$

Second, each block has 8 directions shows in figure 11. The fingerprint ridge's direction is one of them. Then we divide the image into $M \times M$ blocks. In this paper, the value of $M$ is 8 .We use Sobel operators to calculate each pixel's gradient value in horizontal $\left(G_{x}\right)$ and vertical $\left(G_{y}\right)[14]$.The degree of angle $(\theta)$ is obtained by formula (7).

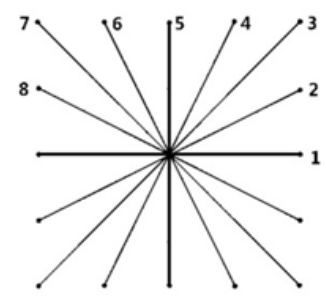

Figure 11. 8 -direction Template

$$
\theta=\frac{1}{2} \tan ^{-1}\left\{\frac{\sum_{i=0}^{M-1} \sum_{j=0}^{M-1} 2 G_{x}(i, j) G_{y}(i, j)}{\sum_{i=0}^{M-1} \sum_{j=0}^{M-1}\left(G_{x}^{2}(i, j)-G_{y}^{2}(i, j)\right)}\right\}
$$

Third, we divide the Sobel calculation image into a great quantity of $8 \times 8$ blocks with no overlap which means that these blocks constitute the whole image. Depending on the value of every point, we design 8 operators ( $\mathrm{sa} 1, \mathrm{sa} 2, \mathrm{sa} 3, \mathrm{sa} 4, \mathrm{sa} 5, \mathrm{sa} 6, \mathrm{sa} 7, \mathrm{sa} 8$ ) from (8) to (14) which are also having $8 \times 8$ size. We can build the fingerprint orientation map like 
figure 12 in the end. The orientation map is made up by small lines which have different direction.

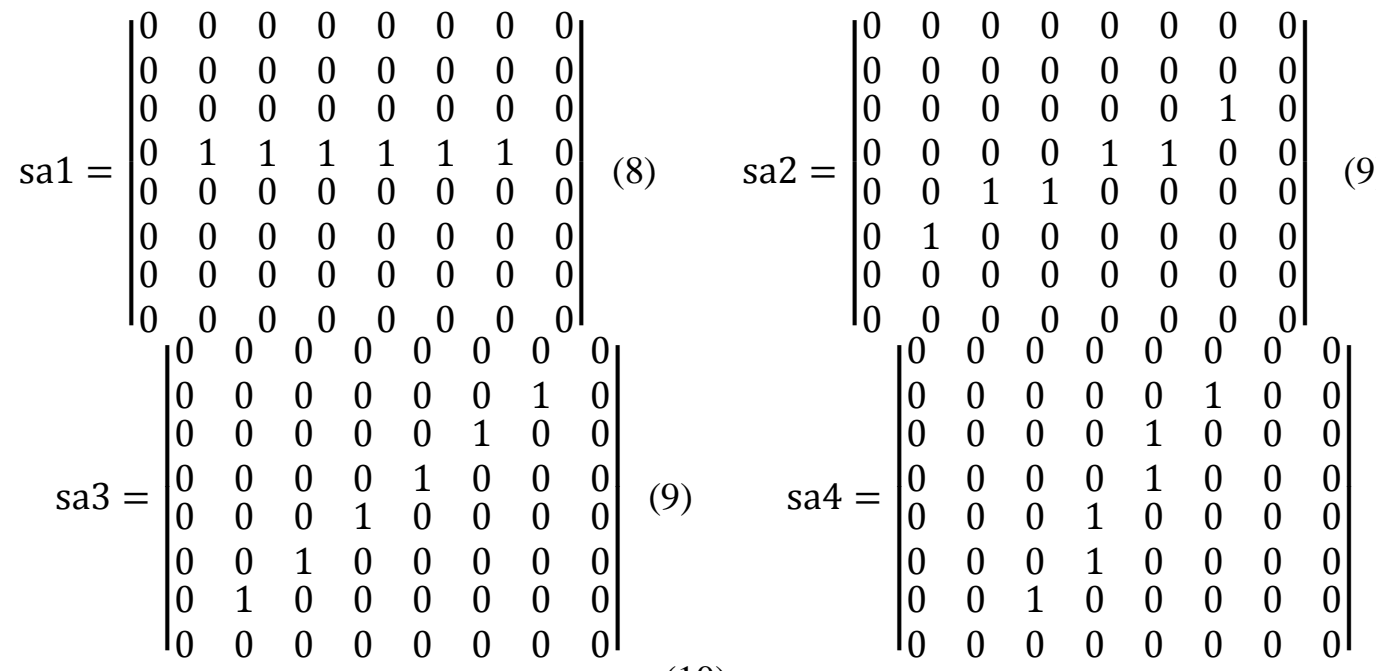

$$
\begin{aligned}
& \operatorname{sa} 5=\left|\begin{array}{llllllll}
0 & 0 & 0 & 0 & 0 & 0 & 0 & 0 \\
0 & 0 & 0 & 1 & 0 & 0 & 0 & 0 \\
0 & 0 & 0 & 1 & 0 & 0 & 0 & 0 \\
0 & 0 & 0 & 1 & 0 & 0 & 0 & 0 \\
0 & 0 & 0 & 1 & 0 & 0 & 0 & 0 \\
0 & 0 & 0 & 1 & 0 & 0 & 0 & 0 \\
0 & 0 & 0 & 1 & 0 & 0 & 0 & 0 \\
0 & 0 & 0 & 0 & 0 & 0 & 0 & 0
\end{array}\right|(11) \quad \text { sa6 }=\left|\begin{array}{llllllll}
0 & 0 & 0 & 0 & 0 & 0 & 0 & 0 \\
0 & 0 & 1 & 0 & 0 & 0 & 0 & 0 \\
0 & 0 & 0 & 1 & 0 & 0 & 0 & 0 \\
0 & 0 & 0 & 1 & 0 & 0 & 0 & 0 \\
0 & 0 & 0 & 0 & 1 & 0 & 0 & 0 \\
0 & 0 & 0 & 0 & 1 & 0 & 0 & 0 \\
0 & 0 & 0 & 0 & 0 & 1 & 0 & 0 \\
0 & 0 & 0 & 0 & 0 & 0 & 0 & 0
\end{array}\right| \\
& \operatorname{sa} 7=\left|\begin{array}{llllllll}
0 & 0 & 0 & 0 & 0 & 0 & 0 & 0 \\
0 & 1 & 0 & 0 & 0 & 0 & 0 & 0 \\
0 & 0 & 1 & 0 & 0 & 0 & 0 & 0 \\
0 & 0 & 0 & 1 & 0 & 0 & 0 & 0 \\
0 & 0 & 0 & 0 & 1 & 0 & 0 & 0 \\
0 & 0 & 0 & 0 & 0 & 1 & 0 & 0 \\
0 & 0 & 0 & 0 & 0 & 0 & 1 & 0 \\
0 & 0 & 0 & 0 & 0 & 0 & 0 & 0
\end{array}\right| \quad(13) \quad \text { sa8 }=\left|\begin{array}{llllllll}
0 & 0 & 0 & 0 & 0 & 0 & 0 & 0 \\
0 & 0 & 0 & 0 & 0 & 0 & 0 & 0 \\
0 & 1 & 0 & 0 & 0 & 0 & 0 & 0 \\
0 & 0 & 1 & 1 & 0 & 0 & 0 & 0 \\
0 & 0 & 0 & 0 & 1 & 1 & 0 & 0 \\
0 & 0 & 0 & 0 & 0 & 0 & 1 & 0 \\
0 & 0 & 0 & 0 & 0 & 0 & 0 & 0 \\
0 & 0 & 0 & 0 & 0 & 0 & 0 & 0
\end{array}\right|
\end{aligned}
$$

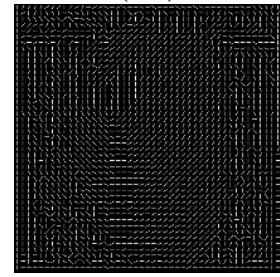

Figure 12. Fingerprint Orientation Map

\section{Step3: The Obtaining of Fingerprint Binary Models}

From the fingerprint orientation map figure 12, we can know that there have irregular directions between fingerprint border and the image background. We need to remove these irregular border directions, and also the background directions for avoiding needless disturbance on the next disposition. In this paper, we use a binary model which shows in the figure 13, the fingerprint part is in write color and the background part in black color to filter out the noises of the orientation map.

The erosion operation of mathematical morphology is used for processing the fingerprint images which were obtained at step 1 . And then we convert the gray images to 
binary images by using a suitable threshold. The Figure 14 is the orientation map filtered by the binary model.

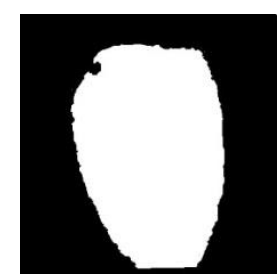

Figure 13. Fingerprint Binary Model

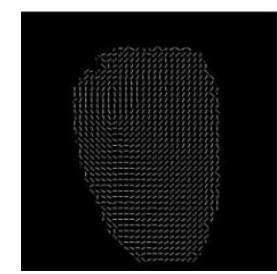

\section{Figure 14. Fingerprint Orientation Map}

\section{Step 4: Searching Singular Points}

In this part, we introduce the algorithm in 3 small steps as follows:

\subsection{Step1: Hit mathematical morphology operation}

In this part, we need to do hit operations using 8 operators on figure 14 . The mathematical morphology operators are designed as se1,se2,se3,se4,se5,se6,se7,se8, shows from (15) to (22).The 8 operators stands for 8 directions respectively. And after the 8 operations, the figure 14 tends into 8 images shows in figure 15 which just reserve the information of the corresponding operator's direction. The form of this information is points.

$$
\begin{aligned}
& \text { se1 }=\left|\begin{array}{llllll}
1 & 1 & 1 & 1 & 1 & 1
\end{array}\right|(15) \quad \operatorname{se} 5=\left|\begin{array}{llllll}
1 & 1 & 1 & 1 & 1 & 1
\end{array}\right|^{T} \\
& \operatorname{se} 2=\left|\begin{array}{llllll}
0 & 0 & 0 & 0 & 0 & 1 \\
0 & 0 & 0 & 1 & 1 & 0 \\
0 & 1 & 1 & 0 & 0 & 0 \\
1 & 0 & 0 & 0 & 0 & 0
\end{array}\right| \quad(17) \quad \operatorname{se} 8=\left|\begin{array}{llllll}
1 & 0 & 0 & 0 & 0 & 0 \\
0 & 1 & 1 & 0 & 0 & 0 \\
0 & 0 & 0 & 1 & 1 & 0 \\
0 & 0 & 0 & 0 & 0 & 1
\end{array}\right| \\
& \text { se3 }=\left|\begin{array}{cccccc}
0 & 0 & 0 & 0 & 0 & 1 \\
0 & 0 & 0 & 0 & 1 & 0 \\
0 & 0 & 0 & 1 & 0 & 0 \\
0 & 0 & 1 & 0 & 0 & 0 \\
0 & 1 & 0 & 0 & 0 & 0 \\
1 & 0 & 0 & 0 & 0 & 0
\end{array}\right| \text { (19) se7 }=\left|\begin{array}{llllll}
1 & 0 & 0 & 0 & 0 & 0 \\
0 & 1 & 0 & 0 & 0 & 0 \\
0 & 0 & 1 & 0 & 0 & 0 \\
0 & 0 & 0 & 1 & 0 & 0 \\
0 & 0 & 0 & 0 & 1 & 0 \\
0 & 0 & 0 & 0 & 0 & 1
\end{array}\right| \\
& \text { se4 }=\left|\begin{array}{llll}
0 & 0 & 0 & 1 \\
0 & 0 & 1 & 0 \\
0 & 0 & 1 & 0 \\
0 & 1 & 0 & 0 \\
0 & 1 & 0 & 0 \\
1 & 0 & 0 & 0
\end{array}\right|(21) \quad \text { se6 }=\left|\begin{array}{llll}
1 & 0 & 0 & 0 \\
0 & 1 & 0 & 0 \\
0 & 1 & 0 & 0 \\
0 & 0 & 1 & 0 \\
0 & 0 & 1 & 0 \\
0 & 0 & 0 & 1
\end{array}\right|
\end{aligned}
$$




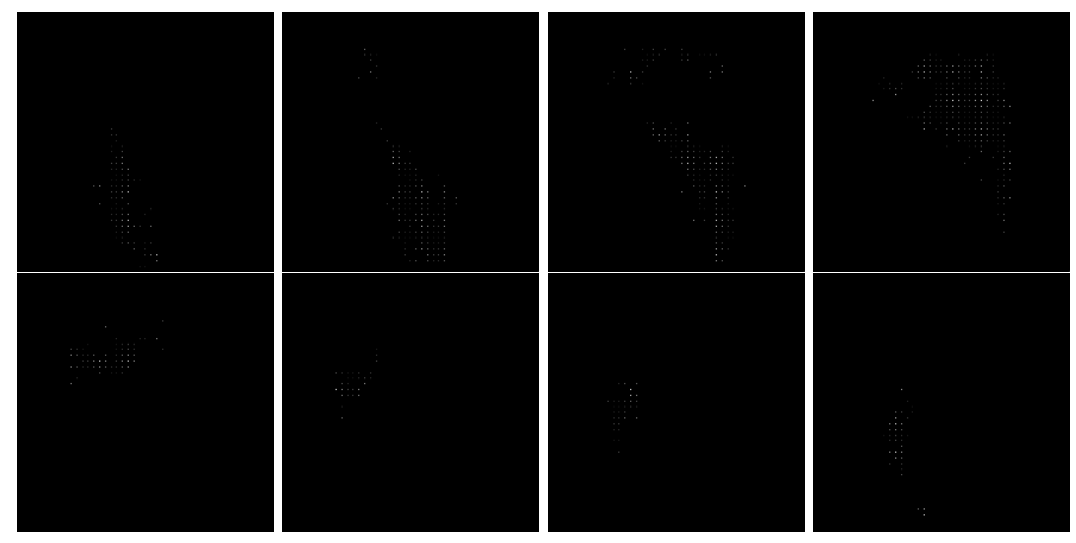

Figure 15. The Hit-operation Images

\subsection{Step2: Dilation Mathematical Morphology Operation}

Then use the 6 diameter disk operators to do dilation operations on the 8 orientation images in Figure 16. The results show as follows:
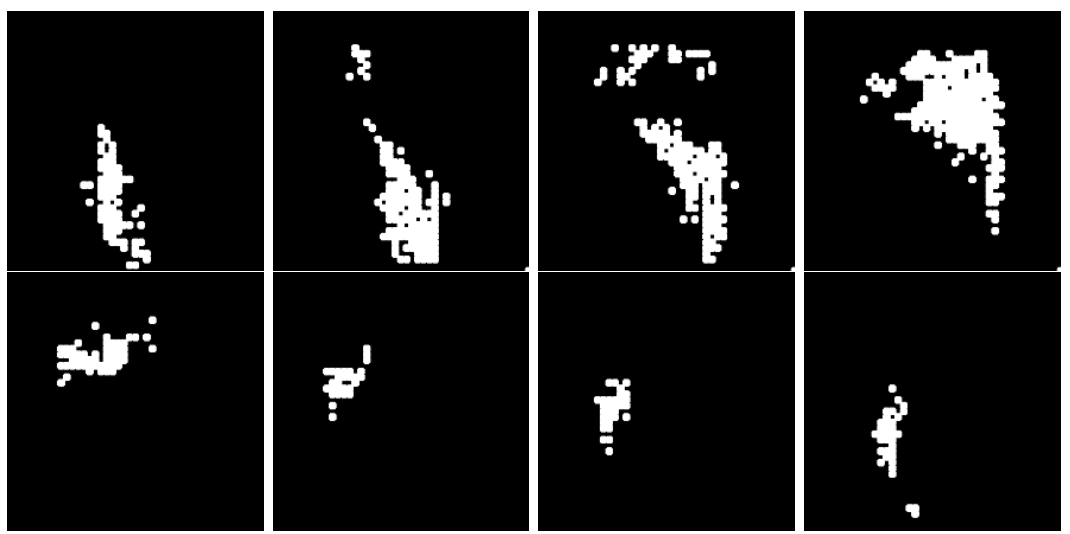

Figure 16. The Dilation Image

We can know in the Figure 15, the original points are independent and not connected with each other. But in the figure 16, some of the points are connecting with each other.

\subsection{Step3: Obtain the Singular Point}

Through the above operation, we get 8 images. Adhere to the 8 possible symmetry axes shows in figure 11, we select images in groups to do AND operation. Each group has 3 images. We first confirm the symmetry direction and choose the image in this direction, and then we choose the two other images whose direction just symmetry to each other with the symmetry direction. The operating groups are defined detailed as follows:

1) The order of the symmetry axis is $3,4,5,6,7,1,8$.

In each axis of symmetry, the 3 images we get from last step need to do and operation. These 3 images includes the 2 images whose direction that symmetry with each other about the axis and 1 image whose direction just at the symmetry direction. The figure 17 shows the five main groups.

Each groups we can obtain 3 images. Some of these images have points, some of them have nothing. If the former group is already having two points, the remnant groups need not to do any operation. If not, the other image groups also need to do and operation.

As we know the Singular point is just one or two, so we need to do further processes. 


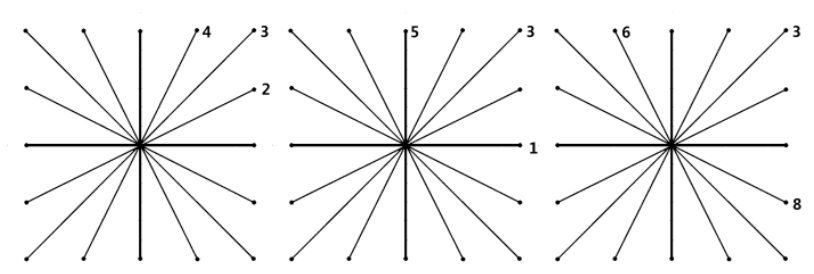

a) Group One

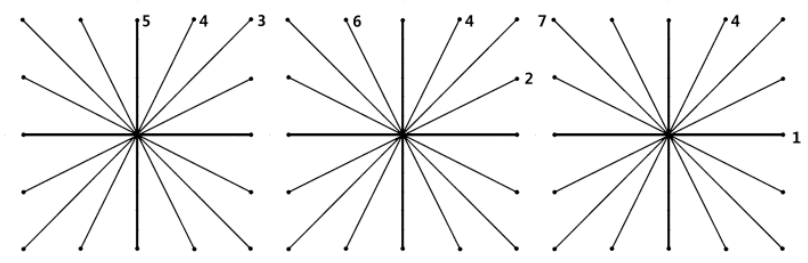

b) Group Two

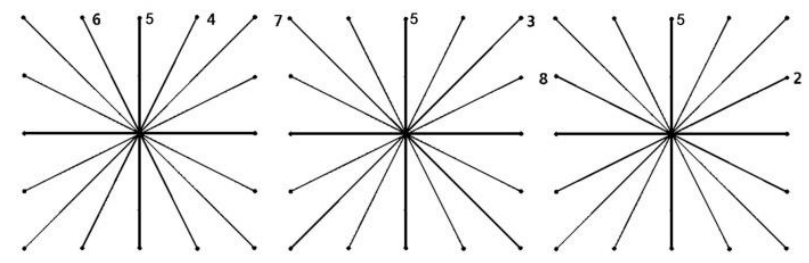

c) Group Three

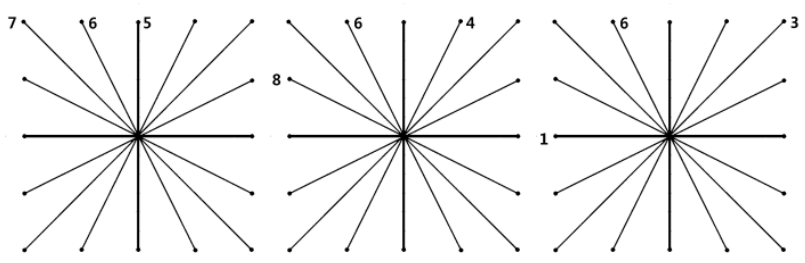

d) Group Four

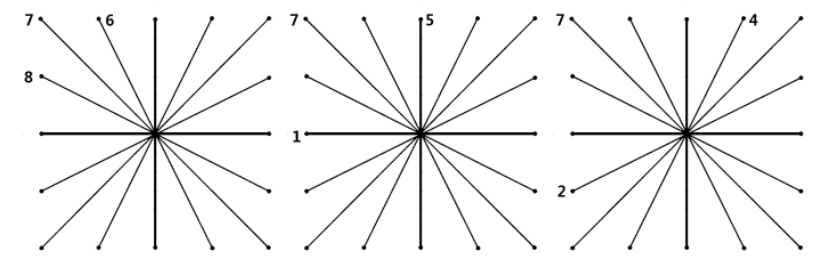

e) Group Five

Figure 17. Five Examples of Direction Groups

2) Check each group's 3 results, if the number of the points is larger than 0 and smaller than 3 then keep the image. Otherwise, delete the image. Apparently, not each group will appear cross point.

3) Making remaining images do and operation. If the image has 1 point, the point is just the core point. If the image has 2 point which shows in the figure 18 , the core point can be obtained by using the following formula.

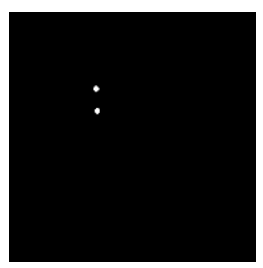

Figure 18. The Result of This Algorithm 
Assume the Coordinate value of these 2 points are $\left(x_{1}, y_{1}\right),\left(x_{2}, y_{2}\right)$. The core point is defined as $\mathrm{O}$ whose coordinate value is $\left(x_{o}, y_{o}\right)$.So the value of $\mathrm{O}$ can be obtained by the formula (23). The center point in the skeleton fingerprint image shows as figure 19.

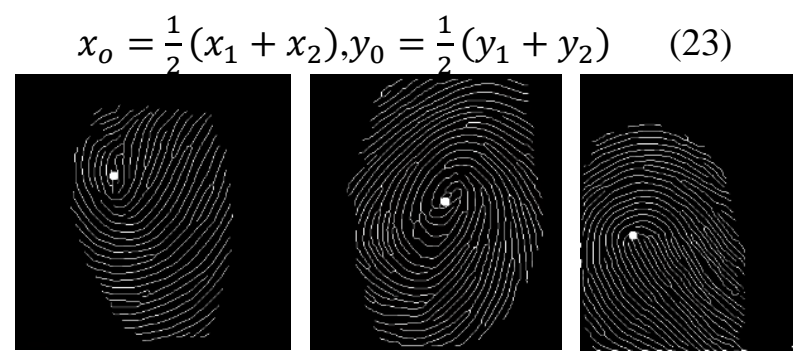

Figure 19.The Center Point in Fingerprints

\section{The Experimental Results}

In order to verify the effect of the algorithm in this paper, we use two parameters as the evaluation criterion- the missing detection rate and the error detection rate [15]. The meaning of missing detection rate is that the fingerprint has the singular points but the algorithm doesn't search them. The meaning of the error detection rate is that the singular points the algorithm search for have the wrong information.

In this paper, we use MATLAB software whose version is e2007 as a development environment and which is running on an Asus notebook computer. The computer has an Asus motherboard -A6VC, an Intel Pentium processor - 1.7G Hz, a 512M Memory.

The experimental images gain from FVC-2002's 4 databases: DB1_A, DB2_A, DB3_A, and DB4_A. The results are shown in the following table.

\begin{tabular}{c|c|c|c|c}
\hline & DB1_A & DB2_A & DB3_A & DB4_A \\
\hline The missing detection rate $(\%)$ & $3.32 \%$ & $7.93 \%$ & $5.23 \%$ & $7.66 \%$ \\
\hline The error detection rate $(\%)$ & $2.45 \%$ & $5.54 \%$ & $4.34 \%$ & $4.38 \%$ \\
\hline
\end{tabular}

Figure 20. The Test of This Algorithm by Two Parameters

From the figure 20, we can know that the fingerprint database with poor quality images have the higher missing rate and false detection rate, like: DB2_A, DB4_A. The fingerprint database with high quality images have lower detection rate and false detection rate, like: DB1_A, DB3_A. But totally, the singular points obtained in this paper can meet requirements of fingerprint's point pattern matching algorithm fully.

\section{Conclusions}

This paper presents a new method to finding the fingerprint image's singular points. After a series steps, we can gain the information of the singular points. The algorithm is simple to understand and tend into truth. Especially it has the advantages that fast in speed and high in accurate and still has a good robustness for the fingerprint's translation and deformation. But deficiencies in this method are also obvious that the accuracy is easy to be influenced by some big noises in the orientation map.

\section{References}

[1] A K. Jain, S. Prabhakar and L. Hong, "A multichannel approach to finger-print classification", IEEE Transactions on Pattern Analysis and Machine Intelligence, vol. 21, (1999), pp. 348-359. 
[2] J. Liu and J. Huang, "Thinning and calculation of center point with on-line fingerprint verification system”, JOURNAL OF HARBIN IN Sn TUTE OF TECHNOLOGY, vol. 32, no. 6, (2000), pp. 91-95.

[3] Y. Mei and G. Cao, “A New Method for Rapid Detection of Fingerprints' Singular Points", CHINESE JOURNAL OF COMPUTERS, vol. 32, no. 5, (2009), pp. 1037-1044.

[4] J. Jia, L. Cai, P. Lu and X. Liu, "Fingerprint matching based on weighting method and the SVM", Neuro computing, no. 70, (2007), pp. 849-858.

[5] W. Sheng, G. Howells, M. C. Fairhurst, F. Deravi and K. Harmer, "Consensus fingerprint matching with genetically optimized approach”, Pattern Recognition, no. 42, (2009), pp. 1399-1407.

[6] P. Ke and Q. Lin, "Fingerprint Singular Points Detection Algorithm Based on Flow Field and Laurent Series", Computer Engineering, vol. 39, no. 6, (2013), pp. 227-235.

[7] M. Kass and A. Witkin, "Analyzing oriented patterns", Computer Vision, Graphics, and Image Processing, vol. 37, no. 3, (1987), pp. 362-385.

[8] Y. Hao and F.-q. Wang, "Fingerprint singular point location based on DFT of orientation field and corner detector", CHINESE JOURNAL OF QUANTUM ELECTRONICS, vol. 26, no. 5, (2009), pp. 518-524.

[9] M-C. Liu, "Wavelet analysis and its application", Publishing House of Qinghua university, BEIJING, (2013).

[10] H. Maitre, "Le traitement des images", Publishing House of Electronics Industry. BEIJING, (2006).

[11] Y. Min, "Digital image processing", China Machine Press, BEIJING, (2005).

[12] P. Soille, "Morphological Image Analysis Principles and Applications Second Edition", (2004).

[13] L.-G. Xu and W. Xu, "Material Evidence Technical, China Renmin UP. BEIJING, (1990).

[14] X. Yang, H. Yang and X. Yang, "Study and Realization of Algorithms of Fingerpirnt Orientation Map", Journal of Southwest University (Natural Science Edition), vol. 30, no. 8, (2008), pp. 142-146.

[15] L. Hang, K. Pang and W. Chui, "Fast Detection Method of Center of the Fingerprint Based on Sample Orientation Field", LASERNAL, vol. 35, no. 3, (2014), pp. 35-36. 